\title{
Quo Vadis Urogynecology 2020 - Innovative Treatment Concepts for Urinary Incontinence and Pelvic Organ Prolapse
}

\section{Quo vadis Urogynäkologie 2020 - innovative Behandlungskonzepte von Harninkontinenz und Genitalprolaps}

\section{(ㄷ) (i) (오) $\ominus$}

\author{
Author \\ Gert Naumann
}

Affiliation

Frauenklinik, Helios Klinikum Erfurt, Universitätsfrauenklinik, Heinrich-Heine-Universität Düsseldorf, Düsseldorf, Germany

\section{Key words}

stress incontinence, suburethral sling, pelvic organ prolapse, sacrocolpopexy, vaginal uterine fixation

Schlüsselwörter

Belastungsinkontinenz, suburethrale Schlinge, Genitaldeszensus, Sakrokolpopexie, vaginale Uterusfixation

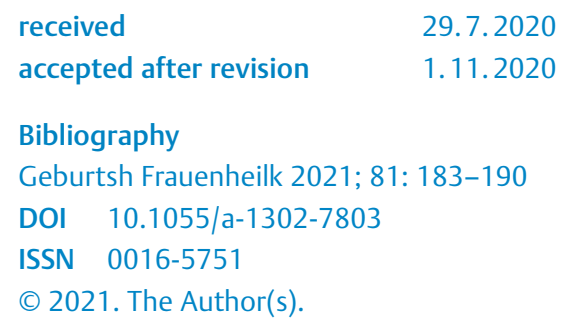

This is an open access article published by Thieme under the terms of the Creative Commons Attribution-NonDerivative-NonCommercial-License, permitting copying and reproduction so long as the original work is given appropriate credit. Contents may not be used for commercial purposes, or adapted, remixed, transformed or built upon. (https://creativecommons.org/licenses/by-nc-nd/4.0/)

Georg Thieme Verlag KG, Rüdigerstraße 14,

70469 Stuttgart, Germany

\section{Correspondence}

Priv.-Doz. Dr. med. habil. Gert Naumann

Frauenklinik, Helios Klinikum Erfurt, Universitätsfrauenklinik, Heinrich-Heine-Universität Düsseldorf

Nordhäuser Straße 74, 99089 Erfurt, Germany

gert.naumann@helios-gesundheit.de

Deutsche Version unter:

https://doi.org/10.1055/a-1302-7803

\section{ABSTRACT}

The current treatment for urinary incontinence and pelvic organ prolapse includes a wide range of innovative options for conservative and surgical therapies. Initial treatment for pelvic floor dysfunction consists of individualized topical estrogen therapy and professional training in passive and active pelvic floor exercises with biofeedback, vibration plates, and a number of vaginal devices. The method of choice for the surgical repair of stress urinary incontinence consists of placement of a suburethral sling. A number of different methods are available for the surgical treatment of pelvic organ prolapse using either a vaginal or an abdominal/endoscopic approach and autologous tissue or alloplastic materials for reconstruction. This makes it possible to achieve optimal reconstruction both in younger women, many of them affected by postpartum trauma, and in older women later in their lives. Treatment includes assessing the patient's state of health and anesthetic risk profile. It is important to determine a realistically achievable patient preference after explaining the individualized concept and presenting the alternative surgical options.

\section{ZUSAMMENFASSUNG}

Die aktuelle Behandlung von Harninkontinenz und Genitalprolaps umfasst vielfältige innovative Möglichkeiten konservativer und operativer Therapien. Eine angepasste lokale Estrogenisierung und professionelle Techniken des passiven und aktiven Beckenbodentrainings mit Biofeedback, Rüttelplatte und verschiedenen Vaginaldevices stellen den Behandlungsbeginn bei Beckenbodenfunktionsstörungen dar. Methode der Wahl zur operativen Sanierung der Belastungsinkontinenz ist die Einlage einer suburethralen Schlinge. Die operative Behandlung des Genitalprolaps bietet verschiedene Methoden auf vaginalem oder abdominal/endoskopischem Weg unter Eigengewebsrekonstruktion oder Nutzung von alloplastischem Material. So gelingt eine individuelle optimale Rekonstruktion sowohl bei der jungen Frau mit zumeist post- 
partalen Schädigungen bis hin zur betagten Patientin in der letzten Lebensphase. Dazu gehört die Einschätzung des gesundheitlichen Zustandes der Patientin und dem bestehenden anästhesiologischen Risikoprofil. Wichtig ist die Ermitt- lung des realistischen Patientenwunsches nach Erläuterung eines individuellen Konzeptes und Darstellung auch alternativer operativer Möglichkeiten.

\section{Introduction}

This year, the COVID-19 pandemic has almost completely eclipsed many, predominantly benign disorders. Particularly older and more vulnerable patients are currently shying away from visiting their GP or attending outpatient clinics to receive the necessary treatment for pronounced pelvic floor dysfunction, even though some patients are dealing with serious problems which are reducing their quality of life.

This article aims to motivate all colleagues to direct affected women to seek appropriate and adequate treatment and encourage their proactive cooperation. The aim of this paper is to provide an update on the different methods available to treat urinary incontinence and pelvic organ prolapse and present the risks involved and the chances of success.

With a prevalence of 30-35 percent in all women from the age of 50 years and above, pelvic floor disorders with pelvic organ prolapse and urinary incontinence are among the most common disorders in women and are associated with serious restrictions of everyday life and a reduced quality of life and sexual health.

The female pelvic floor must fulfil two complex but contrary purposes; outside pregnancy and delivery, it functions as a firm caudal end of the abdominal cavity with regulated evacuation functions and a flexible sexual organ. Complex biochemical changes occur during pregnancy and in childbirth which enable tissues to become extraordinarily flexible and elastic but also to largely recover their previous condition post partum. This double function may lead to an increasing impairment of bladder function in later life with the loss of regulated muscular fixation and connective tissue anchoring and, consequently, displacement of the vagina, bladder or rectum outside the pelvic cavity. The urethral closure mechanism also becomes weaker following the decrease in hormone activity; the consequence can be stress urinary incontinence with involuntary loss of urine when coughing or sneezing or during physical activities. The symptoms associated with overactive bladder have a particularly severe impact on quality of life as patients suffer from urinary urgency and/or urinary urge incontinence.

An individualized, differentiated, diagnostic investigation is essential for the successful treatment of such complex disorders of pelvic floor function as urinary incontinence and pelvic organ prolapse. Recording all distressing symptoms and assessing the patient's psychological stress together with a urogynecological examination which includes various function tests are easy to carry out and are not associated with any major technical costs.

\section{Current Treatment for \\ Stress Urinary Incontinence}

Treatment for stress urinary incontinence largely consists of surgical procedures, carried out either after unsuccessful conservative therapy or to complement conservative treatment. With an overall success rate of around $80 \%$, conservative measures before and after surgery such as behavioral changes (weight loss, avoiding lifting and carrying heavy loads, stool regulation, bladder, training, etc.) and physiotherapy exercises under professional guidance to strengthen the pelvic floor muscles can improve compliance and preserve the results of successful surgery over the long term. The current trend is to provide individual support to improve pelvic floor contractility using innovative biofeedback devices with an optical and/or acoustic signal to monitor results, external exercises carried out on a vibration plate (Galileo training devices), and magnetic field therapy or electrostimulation therapy with external adhesive electrodes to strengthen passive muscle force.

Topical vaginal estrogen application using an estriol preparation improves the general metabolic status of the vaginal epithelium and is effective at reducing the rate of urinary tract infections and the symptoms of urinary urgency. Despite ongoing discussions about the potential negative systemic effects and an overall lack of data, there are enough good arguments to still support offering low-dose topical estriol therapy in addition to hormonefree topical ointment or laser therapy [1].

Drug treatment with duloxetine can reduce the incontinence rate by up to $50 \%$ and should be particularly used for patients who are not able to turn to surgery as an option. In these cases, it is important to ensure that the dosages are gradually increased and subsequently tapered off because of potential intolerances.

Conservative therapy is very promising and usually completely reversible; it is not associated with strong side-effects or serious complications. If conservative treatment does not lead to the desired result, it will at least show the patient how complex and serious the disorder is and will help the affected patient to accept an improvement of $80-85 \%$ following surgery more easily than attempting to guarantee $100 \%$ success following surgery.

The current gold standard of treatment still consists of placement of a tension-free vaginal sling using a retropubic (TVT procedure) or transobturator approach (TVT-O, TOT procedure). This offers excellent results, a continence rate of up to $85 \%$ even after 5 years, and acceptable side-effects and risks.

Compared to other established procedures such as intraurethral and periurethral injections or colposuspension, this type of surgical procedure has significantly better long-term results with lower overall morbidity rates and a better outcome [2]. 
The method of choice for sling placement to repair urinary continence is still the retropubic approach. Numerous studies on its efficacy, recent long-term results covering a period of 17 years, RCTs comparing it with other systems, and the existing register of complications have made sure that physicians are able to offer an effective and safe procedure.

In the author's opinion, transobturator procedures (TOT, TVT-O) offer no significant benefits. The success rates are similar to those obtained with TVT procedures. Complications are also possible due to blind passage of the needles, and while they only occur below the level of the pelvic floor, they are often difficult to treat if they are in the obturator or adductor area. Typical complications of TOT procedures include lacerations of the vaginal sulcus and often significant dyspareunia and pain syndromes, which are less pronounced with retropubic procedures.

A recent meta-analysis of the long-term success rates for both approaches (follow-up $>5$ years) which looked at 5592 papers from 2000 to 2016 evaluated a total of 11 RCTs $(0.6 \%)$ and 5 non-RCTs (0.3\%) [3]. Both approaches had comparable objective and subjective success rates (TVT: $61.6 \%$ obj., 76.5\% subj.; TOT: $64.4 \%$ obj., $81.3 \%$ subj.). The analysis also found no significant differences in the numbers of complications (TVT vs. TOT [OR: 0.83; 95\% Cl: 0.54-1.28]).

Single-incision sling (SIS) procedures began to be developed at the end of the 1990s. They are even less invasive as they only require a single access point. This new approach aimed to significantly reduce the blind passage of needles, because placement of the sling does not involve blind passage through either the retropubic space or the obturatur foramen. Moreover, the sling used for SIS is significantly shorter $(6.5-12 \mathrm{~cm})$, meaning that less alloplastic material is used. If placed correctly with slightly more tension, single-incision slings are a good option to obtain high continence rates with significantly lower invasiveness while avoiding the retropubic region.

A recent meta-analysis (29 RCTs with 3000 patients) showed comparable medium-term continence rates for SIS using established suburethral sling procedures once the now obsolete TVTSecur procedure with its high failure rates was excluded (OR: $0.67,95 \% \mathrm{Cl}: 0.44-1.60)$. SIS was found to be associated with shorter operative times, less blood loss and less pain. No longterm results are available yet [4]. Further studies are not expected at present, as single-incision slings have been reclassified as a hazard class III device based on a warning by the FDA and the new MDR regulations and may currently only be used under study conditions. Many suppliers are therefore withdrawing from the market.

An investigation into the prevalence of surgical urinary incontinence procedures in Germany reported a dramatic decrease of $37 \%$ in the use of suburethral slings between 2010 and 2018 without a compensatory increase in the use of other procedures [5].

Internationally, there has also been a decline in the use of slings and meshes in urogyecology, not least because of the ongoing discussion about the risks and benefits of alloplastic materials in the pelvic floor area, encouraged less by convincing medical arguments than by the dubious practices associated with medical lawsuits in the USA.
Colposuspension, particularly using a laparoscopic approach, is becoming more important again. The Cochrane analysis reported continence rates of $68.9-88 \%$ at 12 months following open surgery, which decreased to $70 \%$ after 10 years and to $65-70 \%$ after 20 years [6]. The laparoscopic approach showed comparable results at 18 months with longer operating times but lower morbidity rates, fewer postoperative complications, and less pain [7]. The many different approaches used in the studies (sutures vs. staples, 1 suture vs. 2 sutures, open colposuspension vs. laparoscopic colposuspension, colposuspension vs. sling) make it difficult to arrive at any robust conclusion. Moreover, endoscopic techniques require skills which have to be specially learned in training programs over a number of years.

Intraurethral injections to strengthen mucosal coaptation and improve continence are also beginning to be used more frequently. Previously used as a second-line therapy, a number of randomized studies have investigated its use in a primary setting. The percentage of patients showing a significant improvement in urinary continence is high but the percentage with complete incontinence is still around $50 \%$. The inferiority of this method in terms of poorer continence rates is compensated by the lower side effects and the avoidance of alloplastic materials [8], although the modern substances used in the injections are also synthetic.

The 2020 results of a randomized study from Finland which compared retropubic TVT sling with Bulkamid injection (polyacrylamide) to treat primary stress incontinence were eagerly awaited [9].

Significant differences in subjective (satisfaction according to the visual analogue score: TVT 95\%, Bulkamid 59.8\%) and objective continence rates (negative cough test: TVT 95\%, Bulkamid $66.4 \%$ ) were found at follow-up after 12 months. There were more complications and more re-operations in the TVT group. The authors recommend that intraurethral injections be offered as an alternative primary treatment.

The use of an artificial sphincter may also be considered, particularly in patients with recurrence. Recent data reported continence rates of $61-100 \%$, but also an increased rate of complications (bladder neck injury: 0-43\%, explantation: 0-45\%, erosion: 0-22\%, mechanical failure: 0-44\%) [10].

\section{Current Treatment for Symptoms of Urinary Urgency with/without Incontinence/Overactive Bladder}

The established first-line therapy for urinary urgency used since many years is conservative treatment with behavioral training and the application of topical estrogen. Second-line therapy includes drug therapy (anticholinergics, $\beta$-3 agonists); in refractory cases or following the failure of treatment, the third-line option is an intravesical Botox injection or sacral neuromodulation.

In gynecology, Botulinum Toxin A injections are usually only administered to women with a non-neurogenic idiopathic overactive bladder. Urinary urgency is treated with 100 units of Botulinum Toxin A. The literature reports high success rates with mod- 
erate side effects such as an increase in the amount of residual urine and the number of urinary tract infections [11].

Based on recent data, modifications to the treatment cascade have been proposed, with Botulinum Toxin injections already used as a second-line treatment. A recent meta-analysis comparing the oral drug Mirabegron with Botulinum Toxin A confirmed the benefits of the Botulinum Toxin injection in terms of fewer episodes of urinary incontinence and a decreased frequency of urination in carefully selected patients [12].

Sacral neuromodulation consists of the surgical implantation of a device which modulates the peripheral sacral nerves, particularly the central signaling pathways. It is used to treat idiopathic or therapy-resistant disorders such as urge incontinence, urinary retention, fecal incontinence, constipation, or chronic pelvic pain syndrome. The device influences the peripheral afferent and efferent nerve fibers of the sacral plexus as well as the sympathetic and parasympathetic nerve fibers of the inferior hypogastric plexus and the preganglionic parasympathetic motor neurons of the sacral segments of the spinal cord as well the somatosensory fibers of the pudendal nerve.

As the devices have become more and more technically sophisticated (device is MRI-compatible, the stimulator is rechargeable), recent studies have confirmed the efficacy and safety of this approach. A recent meta-analysis of 21 studies reported that the method is highly effective with a more than $50 \%$ improvement of incontinence episodes of between $29-76 \%$ and a dryness rate of $43-46 \%$ [13].

Patients must be treated in a pelvic floor center specializing in neuromodulation to ensure long-term success, as treatment involves a complex process, beginning with the correct diagnosis, and must include professional follow-up of treated patients.

\section{Current Treatment for Pelvic Organ Prolapse}

As pelvic organ prolapse is associated with a chronic muscle weakness, the transition between prolapse stages up to and including pronounced pelvic organ prolapse is fluid and patients may not always feel much discomfort.

Mild prolapse of the uterus or vaginal wall with no additional symptoms (no residual urine, no disorders of micturition, no foreign body sensation, no pain, no infections) does not automatically require treatment. However, given the fact that prolapse often progresses, patients should be encouraged to try prophylactic measures (lifestyle changes, avoid lifting and carrying heavy loads, stool regulation, etc.).

Even in cases with pronounced pelvic organ prolapse, patients should first be treated conservatively. This is particularly the case when patients have moderate prolapse or patients are not seriously affected. This also applies to so-called prophylactic surgery ("it could get worse", "you might be too old for surgery later on"). Younger women with mild prolapse often only need supportive measures when they do sports or other forms of physical activity. Even patients unwilling to have surgery and older patients with multiple morbidities can benefit from non-surgical measures.

Conservative therapy including topical estrogen application, repositioning of the prolapse using modern silicon pessaries, and
- Table 1 Factors affecting the choice of surgical approach

in prolapse surgery.

- The patient's state of health; merely considering the patient's age is not sufficient

- Existing anatomical and functional defects in the pelvic floor area

- Intraoperative and postoperative risks of the chosen surgical method

- The success rates of specific methods

- Is the patient still sexually active?

- The patient's wishes and needs in terms of outcome and quality of life

- The physician's technical skill and experience in carrying out specific surgical techniques

professional pelvic floor training can help to stabilize pelvic structures.

Repositioning of the prolapse with the help of a pessary may also uncover hidden incontinence and is therefore an important diagnostic aspect of surgical planning. Pessaries usually serve to bridge the time to surgery but can also be used for longer periods without any problems. To avoid ulcerations, pessary use should always be accompanied by topical hormone treatment and regular checks are necessary.

There are many different surgical strategies used to rectify vaginal or uterine prolapse. Fixation of the prolapsed vaginal vault or uterus can be done using an abdominal or vaginal approach. The focus here is on ensuring sufficient anchoring of the middle vaginal compartment to the supporting structures of the lesser pelvis. For many years, vaginal prolapse surgery used a vaginal approach, but this has changed in recent years and the number of surgical options has increased significantly.

Surgical treatment of pelvic organ prolapse requires a detailed examination of the patient and a carefully weighing up of different aspects, including the choice of the most appropriate surgical method and the best approach ( $\triangleright$ Table 1 ).

\section{Hysterectomy versus Hysteropexy for Prolapse Repair}

In the overwhelming majority of prolapse operations, simultaneous hysterectomy is not indicated. As clearly stated in the current S3-guideline on hysterectomy procedures, extirpation of the uterus during prolapse surgery must be specifically indicated, which is usually not the case ( $\bullet$ Table 2 ) [17].

Uterine preservation is only contraindicated in very specific cases [18].

Preserving the uterus/cervix reduces the rate of surgical suspension failure. Recent randomized studies have shown that fixation combined with uterine preservation is superior even at follow-up after 5 years ( $87 \%$ success rate with sacrospinous hysteropexy vs. $76 \%$ success rate with vaginal hysterectomy and uterosacral ligament fixation) [19]. This has been confirmed in recent meta-analyses which showed equivalent outcomes with lower 
- Table 2 Contraindications for uterus-preserving prolapse repair [17].

- Symptomatic myomas, adenomyosis, endometrial abnormalities

- Recent or previous cervical pathology

- Abnormal or postmenopausal bleeding

- Tamoxifen therapy

- Familial BRCA 1 and 2 risk

- Status post hereditary nonpolyposis colorectal cancer with $40-50 \%$ lifetime risk of endometrial cancer

- Regular gynecological follow-up not assured

operating times and fewer complications if the uterus was preserved [20].

In recent years, various insertion aids were developed for the minimally invasive, tension-free fixation of the cervix to both sacrospinous ligaments. A narrow synthetic band, about $1 \mathrm{~cm}$ wide and $8 \mathrm{~cm}$ long, is transfixed to the anterior cervix and extended from the front above the vesicouterine ligament towards the sacrospinous ligament where it is fixed in place using an insertion aid. This achieves excellent and reliable suspension and leaves the cervix accessible for later cytological controls. The anterior compartment which is also usually involved can be repaired using a fascial graft. The limited alloplastic materials used are not in contact with vaginal tissue, making erosion very rare.

This operation has been carried out since 2017 in our hospital and monitored under study conditions (Splentis ${ }^{\circledR}$, Promedon, Cordoba, Argentina). With around 300 interventions performed and a mean operating time of 22 minutes, the procedure has high subjective and objective success rates and almost no intraoperative and postoperative complications.

\section{Vaginal Approach}

A vaginal procedure is still considered the best approach to rectify pelvic floor defects. All of the relevant structures can be reached with this approach, which also offers the option of administering regional anesthesia. Adequate reconstruction is usually possible using well-established fixation techniques and autologous tissue.

Apical fixation of the uterus or vaginal vault to the sacrospinous ligaments results in sufficient stability. However, the procedure results in a slight dorsal shift of the vagina to a lower position rather than the more optimal, slightly higher, physiological S1-2 position.

This surgical method is particularly suitable for older patients with a sufficiently long vaginal vault. It is associated with low morbidity rates and rapid recovery. A vaginal approach is also preferred when treating patients with previous laparotomies. The slight rightward deviation of the new vaginal anchoring which occurs with the unilateral Amreich-Richter procedure has almost no negative impact on patients' sexuality.

The success rate of this method in the international literature is $35-81 \%$; recurrence of cystocele has been reported in up to $25 \%$ of cases [14].
When fixation to the sacrospinous ligaments (SSL) was compared with fixation to the uterosacral ligaments (SUL), no difference in success rates was found at follow-up after 2 years $(60.5 \%$ SSL vs. 59.2\% SUL) [15].

Unfortunately, the standard and routinely performed procedure is often still vaginal hysterectomy with simultaneous anterior and posterior colporrhaphy. Careful analysis of the location of the defect followed by reconstruction the specific defect would be far more effective. Hysterectomy is usually not necessay. Rectification of a small, completely asymptomatic rectocele is often carried out but offers no benefits and should be a thing of the past [16].

The primary focus of surgical repair should be stable fixation of the apical segment. In many cases, achieving sufficient elongation of the anterior and posterior vaginal wall can make anterior or posterior colporrhaphy procedures unnecessary. The decision whether to opt for sacrospinous fixation or fixation using a modified McCall culdoplasty with incorporation and shortening of the uterosacral ligaments is usually based on tissue quality and the extent of prolapse.

\section{Mesh-supported Vaginal Procedures}

According to the current guideline, reconstruction of the anterior compartment through plication of the endopelvic fascia has a cumulative success rate of $63 \%$ (30-100\%), making it a good option for the primary treatment of pelvic organ prolapse [21].

After many years of experience in general surgery in treating abdominal hernias and using synthetic meshes to manage hernias, alloplastic materials have also found their way into urogynecology. Because of the favorable material properties of large pore monofilament polypropylene, this alloplastic material is widely used in the pelvic floor region.

Vaginal surgery with placement of an alloplastic mesh performed by a surgeon with a lot of experience in pelvic floor surgery can still be a very successful option with many benefits and acceptable risks, if the procedure is performed in patients with the right indication and after carefully weighing up all of the benefits and drawbacks. Benefits include shorter operating times and lower morbidity rates.

Meta-analyses have confirmed the efficacy of this approach, which has significantly better anatomical outcomes compared to autologous tissue repair. Subjective improvement and a better quality of life have been reported both with and without mesh use. More re-operations occur because of erosion or de novo incontinence [22].

In view of the ongoing discussions about the benefits and drawbacks and recent FDA warnings, synthetic mesh devices should only be used in cases with recurrence or very pronounced prolapse where the remaining tissue is unstable and/or the patient has suffered levator avulsion [23, 24].

If mesh insertion is combined with simultaneous hysterectomy, the risk of mesh erosion is 5 times higher; it is 6 times higher for inverted T colpotomy [25]. If the procedure includes mesh placement, then preserving the uterus and minimizing the extent of the colpotomy significantly reduces this complication. Particular care must be taken when treating women with immune defi- 
ciency, diabetes mellitus or pronounced hormone deficiency atrophy. Sexually active women must be informed about the potential for dyspareunia.

The current generation of single incision meshes are introduced through a single vaginal incision and placed using special insertion devices directly, under palpatory or optical control of the relevant landmarks, into the medial sacrospinous ligament at the sciatic spine with no blind passage.

The first analysis of single-incision mesh placement (Calistar S, Promedon) in 107 women (98.1\% postmenopausal) with prolapse recurrence $(87 \%)$ or complex primary prolapse (13\%) at follow-up after 18.5 months reported an anatomical success rate of $98 \%$ and $92 \%$ subjective satisfaction rate. With a mean operating time of 38 minutes, there were no intraoperative complications and no incidences of postoperative pain syndrome. Six women had vaginal mesh erosion, and all of them were treated conservatively with topical application of estrogen [26].

\section{Abdominal Approach}

Median suspension of a prolapsed vaginal vault, cervix or uterus using an abdominal approach can consist of sacrocolpopexy, cervicosacropexy or hysterosacropexy with attachment to the longitudinal anterior ligament below the sacral promontory at the S2 level or laterally with fixation to lateral pelvic wall structures. Because of the longer distance, a synthetic implant (prolene, polyvinylidene fluoride [PVDF] etc.) is used in most cases ( $\triangleright$ Table 3) [27].

Endoscopic procedures are modifications of conventional open surgery procedures: all of the important steps in the reconstruction are carried out in the same way as in open surgery [28]. The postoperative success rates for open surgery and laparoscopic procedures are therefore equivalent [29]. Endoscopic procedures require special surgical skills, and the operating time is usually longer. On the other hand, patients benefit from the advantages of laparoscopy which include better cosmetic results, faster recovery times and a shorter hospital stay. Because of the longer operating time, older patients and patients with comorbidities should generally be treated using a vaginal approach.

There is now sufficient data to compare the results of an abdominal approach with those of vaginal surgery. A recent Cochrane analysis of the treatment of apical prolapse compared outcomes after vaginal and abdominal surgery in 600 patients from 6 RCT studies [30]. After abdominal surgery, $7 \%$ of patients still reported a feeling of heaviness in the vagina; the figure for vaginal surgery was $14 \%$ (7-27\%) (RR: 2.11$)$.

Recurrence was more common after vaginal surgery (31-63\% vs. $23 \%$ after sacrocolpopexy, RR: 1.89$)$. Surgery for recurrence was more common following vaginal surgery (5-18\%, RR: 2.28 ) compared to abdominal procedures (4\%). There were no significant differences in the occurrence of stress urinary incontinence after prolapse repair. Dyspareunia was more common in the group treated with vaginal surgery (RR: 2.53).

In recent years, new open and laparoscopic techniques have been developed which do not repair the prolapse through median fixation in the sacral area or promontory but through fixation to the lateral pelvic wall. Possible cases for whom this method would
- Table 3 Various techniques used in abdominal-endoscopic prolapse surgery [27].

- Median suspension of the vaginal vault, cervix or uterus to the longitudinal anterior ligament at the level of the sacral promontory or S1/S2 level

- Lateral anchoring of uterus or vaginal vault to the rectal fascia using a mesh while avoiding the area of the sacral promontory

- Laparoscopic pectopexy with bilateral fixation of the vagina or cervix to the iliopectineal ligaments at the $\$ 2$ level

- Bilateral vaginal fixation using laparoscopic vaginosacropexy (laVASA) or laparoscopic cervicosacropexy (laCESA) with mesh placement, repair of the uterosacral ligaments, and fixation to the prevertebral fascia at the $\mathrm{S} 1$ level

be suitable are obese patients or patients with extensive adhesions which make it difficult to access the middle of the longitudinal anterior ligament. In pectopexy procedures, the iliopectineal ligament is used for lateral fixation as is also the case in colposuspension or lateral repair [31].

The CESA/VASA procedure aims to repair prolapsed uterosacral ligaments and restore them to their physiological position. This is done by bilateral lengthening for a defined length instead of single unilateral median suspension. Elevation of the uterus/vaginal vault and the bladder floor should significantly reduce the urinary urge symptoms. In a recent study, 120 women with prolapse and urinary incontinence had an endoscopic CESA/VASA procedure; in addition to a mean operating time of 88 minutes and a mean patient age of 66 years, urinary urge incontinence was resolved in in $65 \%$ of all cases, with only 4 cases requiring a repeat of apical fixation [32]. However, these data were only subjective, based on the responses to a questionnaire. Both procedures are still in their experimental stages and should be carried out by experienced laparoscopic surgeons in the context of medical studies because of the higher possibility of complications.

New, robot-assisted, minimally invasive surgical systems have now also found their way into gynecological surgery and pelvic floor procedures. The Da Vinci surgical system from Intuitive offers a greater range of motion and dexterity and a higher resolution without movement artefacts, improving precision during dissection. The currently available data on sacrocolpopexy procedures report equivalent success rates and a good safety profile.

New and still experimental surgical techniques should only be used under study conditions. Affected women should be informed in detail about the lack of scientific data, the lack of medium and long-term results, and established alternative techniques.

Because of the complexity of the treatment options, women with pelvic floor dysfunction should be treated by certified specialists in pelvic floor centers. The opportunities for professional interdisciplinary cooperation and the range of expertise should ensure the best possible treatment of a complex and chronic problem. 


\section{Conclusions}

The prerequisite for successful treatment is careful evaluation of the individual defect and the patient's symptoms of urinary incontinence and/or pelvic organ prolapse. This must include assessment of the patient's state of health and anesthetic risk profile.

It is also important to discover the patient's wishes to understand the surgical and conservative treatment options and alternatives in order to offer her a realistic explanation of the individual concepts.

The gold standard for treating stress incontinence remains the placement of a suburethral sling. If this option is not available, a pubovaginal sling procedure, colposuspension or intraurethral injection can be good alternatives.

When carrying out a surgical repair of pelvic organ prolapse, the specific defects, the overall constellation and the patient's specific expectations need to be combined into a personalized concept which explores the different vaginal and abdominal/endoscopic options including autologous tissue reconstruction and the use of alloplastic material.

A vaginal approach is useful for older patients, patients with posterior apical defects, patients requiring fast and non-invasive therapy, and sexually inactive patients. The results are equivalent and offer the benefit of local/regional anesthesia and shorter operating times.

Abdominal and laparoscopic approaches are more suitable for younger women, patients with anterior apical defects, patients who tolerate general anesthesia, and sexually active patients.

Surgical prolapse repair can often be carried out with preservation of the uterus.

\section{Conflict of Interest}

Received study funding and speaker's fees from Promedon.

\section{References}

[1] Crean-Tate KK, Faubion SS, Pederson HJ et al. Management of genitourinary syndrome of menopause in female cancer patients: a focus on vaginal hormonal therapy. Am J Obstet Gynecol 2020; 222: 103-113

[2] Ford AA, Taylor V, Ogah J et al. Midurethral slings for treatment of stress urinary incontinence review. Neurourol Urodyn 2019; 38: S70-S75

[3] Leone Roberti Maggiore U, Finazzi Agrò E, Soligo M et al. Long-term outcomes of TOT and TVT procedures for the treatment of female stress urinary incontinence: a systematic review and meta-analysis. Int Urogynecol J 2017; 28: 1119-1130

[4] Kim A, Kim MS, Park Y] et al. Clinical outcome of single-incision slings, excluding TVT-Secur, vs. standard slings in the surgical management of stress incontinence: an updated systematic review and meta-analysis. BJU Int 2019; 123: 566-584

[5] Huebner M, Tunn R, Reisenauer C et al. Surgical procedures for the treatment of stress urinary incontinence (SUI) in the light of the updated FDA-warning and its effects on practise patterns in Germany between 2010 and 2018. Abstract 63. EUGA 2020 Interactive Meeting 11.12.12.2020 Dublin

[6] Veit-Rubin N, Dubuisson J, Ford A et al. Burch colposuspension. Neurourol Urodyn 2019; 38: 553-562
[7] Freites ], Stewart F, Omar MI et al. Laparoscopic colposuspension for urinary incontinence in women. Cochrane Database Syst Rev 2019; (12): CD002239

[8] Leone Roberti Maggiore U, Bogani G, Meschia M et al. Urethral bulking agents versus other surgical procedures for the treatment of female stress urinary incontinence: a systematic review and meta-analysis. Eur J Obstet Gynecol Reprod Biol 2015; 189: 48-54

[9] Itkonen Freitas AM, Mentula M, Rahkola-Soisalo P et al. Tension-Free Vaginal Tape Surgery versus Polyacrylamide Hydrogel Injection for Primary Stress Urinary Incontinence: A Randomized Clinical Trial. J Urol 2020; 203: 372-378

[10] Peyronnet B, O'Connor E, Khavari R et al. AMS-800 Artificial urinary sphincter in female patients with stress urinary incontinence: A systematic review. Neurourol Urodyn 2019; 38 (Suppl. 4): \$28-S41

[11] Cui $Y$, Zhou $X$, Zong $H$ et al. The efficacy and safety of onabotulinumtoxin $\mathrm{A}$ in treating idiopathic $\mathrm{OAB}$ : A systematic review and meta-analysis. Neurourol Urodyn 2015; 34: 413-419

[12] Lozano-Ortega G, Walker D, Rogula B et al. The Relative Efficacy and Safety of Mirabegron and OnabotulinumtoxinA in Patients With Overactive Bladder who Have Previously Been Managed With an Antimuscarinic: A Network Meta-analysis. Urology 2019; 127: 1-8

[13] Tutolo M, Ammirati E, Heesakkers J et al. Efficacy and Safety of Sacral and Percutaneous Tibial Neuromodulation in Non-neurogenic Lower Urinary Tract Dysfunction and Chronic Pelvic Pain: A Systematic Review of the Literature. Eur Urol 2018; 73: 406-418

[14] Coolen AWM, Bui BN, Dietz V et al. The treatment of post-hysterectomy vaginal vault prolapse: a systematic review and meta-analysis. Int Urogynecol J 2017; 28: 1767-1783

[15] Barber MD, Brubaker L, Burgio KL et al. Comparison of 2 transvaginal surgical approaches and perioperative behavioral therapy for apical vaginal prolapse: the OPTIMAL randomized trial. JAMA 2014; 311: 1023-1034 [published correction in JAMA 2015; 313: 2287]

[16] Sutkin G, Zyczynski HM, Sridhar A et al. Association between adjuvant posterior repair and success of native tissue apical suspension. Am J Obstet Gynecol 2020; 222: 161.e1-161.e8

[17] Neis KJ, Zubke W, Römer T et al. Indications and Route of Hysterectomy for Benign Diseases. Guideline of the DGGG, OEGGG and SGGG (S3 Level, AWMF Registry No. 015/070, April 2015). Geburtshilfe Frauenheilkd 2016; 76: 350-364

[18] Gutman R, Maher C. Uterine-preserving POP surgery. Int Urogynecol ] 2013; 24: 1803-1813

[19] Schulten SFM, Detollenaere RJ, Stekelenburg J et al. Sacrospinous hysteropexy versus vaginal hysterectomy with uterosacral ligament suspension in women with uterine prolapse stage 2 or higher: observational follow-up of a multicentre randomised trial. BMJ 2019; 366: I5149

[20] Meriwether KV, Antosh DD, Olivera CK et al. Uterine preservation vs. hysterectomy in pelvic organ prolapse surgery: a systematic review with meta-analysis and clinical practice guidelines. Am J Obstet Gynecol 2018; 219: 129-146.e2

[21] Baessler K, Aigmüller T, Albrich S et al. Diagnosis and therapy of female pelvic organ prolapse. Guideline of the DGGG, SGGG and OEGGG (S2eLevel, AWMF Registry Number 015/006, April 2016). Geburtshilfe Frauenheilkd 2016; 76: 1287-1301

[22] Maher C, Feiner B, Baessler K et al. Transvaginal mesh or grafts compared with native tissue repair for vaginal prolapse. Cochrane Database Syst Rev 2016; (2): CD012079

[23] Chapple CR, Cruz F, Deffieux X et al. Consensus Statement of the European Urology Association and the European Urogynaecological Association on the Use of Implanted Materials for Treating Pelvic Organ Prolapse and Stress Urinary Incontinence. Eur Urol 2017; 72: 424-431

[24] Wong V, Shek KL, Goh J et al. Cystocele recurrence after anterior colporrhaphy with and without mesh use. Eur J Obstet Gynecol 2014; 172: $131-135$ 
[25] Collinet P, Belot F, Debodinance P et al. Transvaginal mesh technique for pelvic organ prolapse repair: mesh exposure management and risk factors. Int Urogynecol J 2006; 17: 315-320

[26] Naumann G, Hüsch T, Mörgeli C et al. Mesh-augmented transvaginal repair of recurrent or complex anterior pelvic organ prolapse in accordance with SCENIHR-opinion. Int Urogynecol J 2020. doi:10.1007/ s00192-020-04525-9

[27] Wattiez A, Nasir R, Al Maamari B et al. Laparoscopic prolapse surgery: types and evidence. Curr Opin Obstet Gynecol 2016; 28: 430-434

[28] Ganatra AM, Rozet F, Sanchez-Salas R et al. The current status of laparoscopic sacrocolpopexy: a review. European Urol 2009; 55: 1089-1105

[29] Coolen AWM, van Oudheusden AM], Mol BW] et al. Laparoscopic sacrocolpopexy compared with open abdominal sacrocolpopexy for vault prolapse repair: a randomised controlled trial. Int Urogynecol J 2017; 28: $1469-1479$
[30] Maher C, Feiner B, Baessler K et al. Surgical management of pelvic organ prolapse in women. Cochrane Database Syst Rev 2013; (4): CD004014

[31] Noé KG, Schiermeier S, Alkatout I et al. Laparoscopic pectopexy: a prospective, randomized, comparative clinical trial of standard laparoscopic sacral colpocervicopexy with the new laparoscopic pectopexy-postoperative results and intermediate-term follow-up in a pilot study. J Endourol 2015; 29: 210-215

[32] Rexhepi S, Rexhepi E, Stumm M et al. Laparoscopic bilateral cervico-sacropexy and vaginosacropexy: new surgical treatment option in women with pelvic organ prolapse and urinary incontinence. J Endourol 2018; 32: 1058-1064

[33] De Gouveia De Sa M, Claydon LS et al. Robotic versus laparoscopic sacrocolpopexy for treatment of prolapse of the apical segment of the vagina: a systematic review and meta-analysis. Int Urogynecol J 2016; 27: 355-366 